ISSN 0103-5150

Fisioter. Mov., Curitiba, v. 24, n. 4, p. 737-743, out./dez. 2011 Licenciado sob uma Licença Creative Commons

\title{
Avaliação da marcha em ambiente terrestre em indivíduos com síndrome de Down
}

\author{
Gait value in land environments in the \\ individuals with Down syndrome
}

\section{Daniele Cristina do Carmo de Ávila ${ }^{[a]}$, Fernanda Souza Pedro Bom ${ }^{[b]}$, Luciano Magno Juschaks ${ }^{[c]}$, Danieli Isabel Romanovitch Ribas ${ }^{[\mathrm{d}]}$}

[a] Acadêmica do 8o período do curso de Fisioterapia das Faculdades Integradas do Brasil (UNIBRASIL), Curitiba, PR - Brasil, e-mail: danycarmo@ig.com.br

[b] Acadêmica do 8o período do curso de Fisioterapia das Faculdades Integradas do Brasil (UNIBRASIL), Curitiba, PR - Brasil, e-mail: fesbom5@yahoo.com.br

[c] Acadêmico do 8o período do curso de Fisioterapia das Faculdades Integradas do Brasil (UNIBRASIL), Curitiba, PR - Brasil, e-mail: bmmagno@yahoo.com.br

[d] Mestre em Tecnologia da Saúde pela PUCPR, docente do curso de Fisioterapia das Faculdades Integradas do Brasil (UNIBRASIL), Curitiba, PR - Brasil, e-mail: danieliribas@yahoo.com.br

\section{Resumo}

Introdução: Indivíduos portadores da síndrome de Down (SD) apresentam atraso na aquisição dos padrões fundamentais de movimento, tendo como característica básica a dificuldade em formar e selecionar programas motores. Objetivo: Determinar as variáveis lineares da marcha em ambiente terrestre de indivíduos com SD e compará-las com os valores obtidos na literatura para indivíduos hígidos. Métodos: Estudo transversal realizado com 12 indivíduos (ambos os gêneros, 18 anos $\pm 2,8$ ), submetidos à avaliação das variáveis lineares da marcha em ambiente terrestre, por meio da impressão das pegadas em uma passarela de papel pardo de 15 metros de comprimento e 0,6 de largura. Resultados: 0 grupo analisado apresentou tamanho do passo de $(41,62 \pm 8,39)$, tamanho da passada $(83,24 \pm 16,78)$, velocidade da marcha $(0,60 \mathrm{~m} / \mathrm{s} \pm 0,15)$ e cadência de $(89,35 \pm 14,33$ passos/minuto). Conclusão: Os valores obtidos para as variáveis lineares (tamanho do passo e passada, cadência e velocidade da marcha), neste estudo, indicam diferenças com 
os valores encontrados na literatura em relação a indivíduos hígidos, os valores obtidos são menores aos referenciados na literatura.

Palavras-chave: Síndrome de Down. Marcha. Variáveis lineares. Avaliação.

\section{Abstract}

Introduction: Patients with Down syndrome (DS) present a delayed acquisition of fundamental Movement patterns, showing as a basic characteristic a difficulty to form and select motor programs. Objective: This study aimed to determine the variables of linear motion on the ground of patients with DS and to compare them with values of healthy individuals obtained in the literature. Methods: It is a cross-sectional study with 12 subjects (both gender, 18 years \pm 2.8 ) submitted to an evaluation of variables of linear motion on the ground, through the printing of the footsteps on a brown paper footbridge with 15 meters long and 0.6 in width. Results: The analyzed group showed a step size of $41.62 \pm 8.39$, stride length $83.24 \pm 16.78$, motion velocity of 0.60 meters per second \pm 0.15 and a rate of $89.35 \pm 14,33$ steps per minute. Conclusion: The values obtained for the linear variables (step and stride size, cadence and motion speed) in this study indicate differences with the values found in the literature for healthy individuals, the found values are lower than the reported in the literature.

Keywords: Down syndrome. Gait. Linear variables. Evaluation.

\section{Introdução}

A síndrome de Down (SD) é uma alteração do cromossomo de número 21, é a alteração genética mais comum, sendo caracterizada pelos aspectos fenotípicos bem definidos (1). A base cromossômica da SD é clara, porém o motivo da anomalia cromossômica é pouco compreendido (1). 0 diagnóstico pode ser feito por meio da cariotipagem cromossômica, que pode ser realizada durante a gestação, após análise citogenética das vilosidades coriônicas ou células do líquido amniótico (1). Crianças com SD são acometidas por diversas afecções, como: problemas de audição, visão; cardiopatias congênitas; distúrbios da tireoide; problemas neurológicos; alterações da coluna cervical; obesidade; e hipotonia (2). Um dos problemas congênitos que mais prejudica o desenvolvimento, principalmente o desenvolvimento psicomotor da criança com Down, é a hipotonia generalizada, caracterizada por flacidez muscular e lassidão ligamentar (3), dificultando a marcha adequada.

Essa flexibilidade exige a disponibilidade de estratégias motoras múltiplas e a capacidade de selecionar a estratégia adequada, de acordo com as características da tarefa e do ambiente. A incapacidade de adaptar os movimentos às demandas mutáveis da tarefa é uma característica de muitos pacientes com disfunções neurológicas (4).
A maturação do sistema nervoso central (SNC) é um elemento de importância crítica para a evolução motora (5). A marcha é o marco principal do desenvolvimento infantil e é a habilidade motora fundamental para a locomoção (6), sendo dividida em fases e subfases que irão torná-la harmoniosa e funcional, o seu padrão normal estará estabelecido em torno dos 2 aos 3 anos de idade e é marcado por características específicas, que sofrem mudanças à medida que a criança se desenvolve. Contudo, o padrão da marcha adulta será determinado aos 7 anos (7).

A clara importância do desenvolvimento motor para o desenvolvimento integrado do ser humano justifica o acompanhamento de suas etapas, no sentido da detecção precoce de alterações que possam ser minimizadas, a fim de não interferirem no desenvolvimento global (8). Sendo assim, encontram-se relatos de que indivíduos com SD apresentam atraso nas aquisições de marcos motores básicos (9), porém o valor mais significativo encontrado é quanto à deambulação, pois as crianças com SD andam somente após 1 ano e 4 meses de idade (3). Entretanto, também há crianças que iniciaram a deambulação aos 3 anos e meio (10), enquanto a criança hígida adquire a marcha por volta do $12^{\circ}$ ao $15^{\circ}$ mês (11). Isso seria atribuído às alterações do sistema nervoso decorrentes da síndrome, dificultando a produção e o controle de ativações musculares apropriadas (12). 
Estudos mostram que indivíduos portadores da síndrome de Down apresentam características como lentidão, seleção de estratégias não usuais e atraso na aquisição dos padrões fundamentais de movimento, apresentando como dificuldade básica a seleção de programas motores. Esses indivíduos teriam problemas para combinar esses movimentos, recorrendo a estratégias motoras diferentes ou não usuais para solução de um determinado problema motor (12).

Indivíduos com SD recorrem a uma resposta adaptativa como estratégia. Esse padrão aparentemente anormal deve ser visto como um sinal de que o sistema nervoso central está reorganizando suas prioridades para alcançar uma solução motora, dentre as várias permitidas pela redundância do sistema motor (13).

0 conhecimento adequado do padrão de marcha normal deve ser levado em conta já que muitas características atípicas observadas na marcha humana são prováveis resultantes de adaptações, não podendo ser consideradas patológicas (14).

Rítmica e aparentemente sem exigir esforço, a marcha é um evento contínuo que se constitui em transferir o peso de um membro inferior para outro, com o objetivo de se deslocar de um ponto a outro (15). 0 ciclo da marcha é caracterizado por dois contatos iniciais consecutivos realizados pelo mesmo membro inferior, sendo dividido em duas fases distintas (apoio e balanço) (16). Essa sequência de eventos também é definida como passada, que é o período correspondente a um ciclo da marcha (17). Para análise da marcha, algumas variáveis devem ser observadas como tamanho do passo/passada, velocidade e cadência.

Considerando os fatos apresentados, foi observada a necessidade de estudos que identifiquem alterações na marcha de indivíduos com SD, a fim de melhorar protocolos de reabilitação e padrões alterados de marcha. Portanto, este estudo tem como objetivo avaliar a marcha de indivíduos com síndrome de Down em ambiente terrestre, identificando as variáveis lineares (tamanho do passo, passada, cadência e velocidade da marcha) e compará-las com os valores obtidos na literatura para indivíduos hígidos.

\section{Materiais e métodos}

\section{Sujeitos}

Fizeram parte deste estudo transversal 12 indivíduos com síndrome de Down (SD), idade média de
18 anos (DP $\pm 2,8$ ), selecionados em uma escola especializada da cidade de Curitiba, PR.

Os critérios de inclusão foram: indivíduos com marcha independente e que entendessem comando verbal; e os de exclusão: indivíduos com limitações físicas dos sistemas osteomioarticulares, com deficiência visual e auditiva, deficiência mental moderada e grave, uso sistemático de medicação anticonvulsivante, cadeirantes, uso de próteses que auxiliam na deambulação e/ou tivessem associada à síndrome outra patologia neurológica (paralisia cerebral, autismo, síndrome de Rett, síndrome de West).

O presente estudo seguiu a Resolução 196/96 do Conselho Nacional de Saúde e foi aprovado pelo Comitê de Ética em Pesquisa das Faculdades Integradas do Brasil (UNIBRASIL) sob o Parecer 033-01/2009.

\section{Procedimentos}

Após a assinatura do Termo de Consentimento Livre e Esclarecido pelos pais e/ou responsáveis, a amostra do estudo foi submetida à avaliação da marcha em ambiente terrestre. Para essa avaliação foi solicitado ao indivíduo que deambulasse em uma passarela de papel pardo de 15 metros de comprimento e 0,6 de largura, fixada com fita crepe Adelbrás ${ }^{\circledR}$, para que não houvesse deslizamentos ou tropeções no decorrer da avaliação (18).

No início da passarela foi posicionado um recipiente plástico raso com tinta de tecido Acrílex ${ }^{\circledR}$ para que a criança marcasse sua região plantar. 0 objetivo da tinta foi gerar uma marcação nítida dos pés dos indivíduos. Posteriormente foi realizada a análise dos parâmetros: passo, passada, tempo da passada, velocidade da passada e cadência.

Os dados coletados foram registrados em uma ficha de avaliação. Foram calculadas a velocidade e a cadência, levando em consideração a média de 3 passadas, e para o cálculo da velocidade foi utilizada a fórmula $\mathrm{V}=\mathrm{d}(\mathrm{m}) \cdot \mathrm{t}(\mathrm{s})$. Para a cadência foi calculado o número de passos por minutos; e o tempo foi obtido por meio de um cronômetro da marca Timex ${ }^{\circledR}$ Sport T15J581.

O comprimento do passo foi obtido pela distância de uma passada perpendicular em centímetros, da extremidade posterior do calcanhar até a extremidade posterior do calcanhar do outro pé. 0 comprimento da passada foi obtido pela distância perpendicular posterior do calcanhar de um pé até a 
extremidade posterior do mesmo calcanhar. Essas medidas foram obtidas por meio de uma fita métrica da marca ISP.

\section{Análise estatística}

Os dados coletados foram inseridos em planilhas e apresentados por meio de estatísticas descritivas, tabelas com média e desvio-padrão.

\section{Resultados}

Na Tabela 1 são apresentados os valores obtidos em relação às características da amostra, como: média de idade, altura, peso, índice de massa corpórea (IMC) e os respectivos desvios-padrões dos sujeitos.

Na Tabela 2 podem ser visualizados os valores médios obtidos em relação às variáveis lineares avaliadas: comprimento do passo, comprimento da passada, tempo da passada, velocidade e cadência.

Na Tabela 3 são apresentados os valores para comprimento do passo, comprimento da passada, tempo da passada, velocidade e cadência dos sujeitos separados por gênero: masculino $(\mathrm{n}=6)$ e feminino $(\mathrm{n}=6)$. Observa-se que os valores obtidos para comprimento do passo, comprimento da passada e velocidade da passada no grupo feminino são inferiores ao do grupo masculino. Para tempo da passada e cadência os valores do grupo feminino são mais altos.

\section{Discussão}

Adolescentes com SD frequentemente apresentam alterações posturais (19), sobrepeso (20) e déficits de equilíbrio que influenciam no padrão da marcha (19); alterações que estão relacionadas com a instabilidade corporal.

Ao avaliar a marcha de indivíduos com SD em relação às variáveis lineares, na amostra selecionada, foi obtido, para o parâmetro de tamanho do passo, o valor de 41,62 (DP $\pm 8,39$ ), o tamanho da passada de 83,24 (DP $\pm 16,78$ ), o tempo da passada de 14 segundos (DP $\pm 3,4$ ), a velocidade de $0,60 \mathrm{~m} / \mathrm{s}$ (DP $\pm 0,15$ ) e a cadência de 89,35 (DP $\pm 14,33$ ). Segundo Winter; Rose e Gamble; Holden; Chung; Barela, Stolf e Duarte; Guimarães et al.; Ribas et al.; Dini e David
Tabela 1 - Média de idade, altura, peso e IMC dos sujeitos participantes da pesquisa $(n=12)$

\begin{tabular}{lcccc}
\hline & $\begin{array}{c}\text { Idade } \\
(\text { anos })\end{array}$ & $\begin{array}{c}\text { Altura } \\
(\mathbf{m})\end{array}$ & $\begin{array}{c}\text { Peso } \\
(\mathbf{k g})\end{array}$ & $\begin{array}{c}\text { IMC } \\
\left(\mathbf{k g} / \mathbf{m}^{2}\right)\end{array}$ \\
\hline Média & 18,00 & 1,53 & 62,67 & 26,42 \\
DP & $\pm 2,8$ & $\pm 0,11$ & $\pm 15,53$ & $\pm 3,83$ \\
\hline
\end{tabular}

Fonte: Dados da pesquisa.

Tabela 2 - Média dos dados lineares para comprimento do passo, comprimento da passada, tempo da passada, velocidade e cadência dos sujeitos da pesquisa $(n=12)$ obtidos na avaliação

\begin{tabular}{lc}
\hline Comprimento do passo $(\mathrm{m})$ & $41,62( \pm 8,39)$ \\
Comprimento da passada $(\mathrm{m})$ & $83,24( \pm 16,78)$ \\
Tempo da passada $(\mathrm{s})$ & $14,00( \pm 3,4)$ \\
Velocidade da passada (m/s) & $0,60( \pm 0,15)$ \\
Cadência (passos/min) & $89,35( \pm 14,33)$
\end{tabular}

Fonte: Dados da pesquisa.

Tabela 3 - Média dos dados lineares para comprimento do passo, comprimento da passada, velocidade e cadência dos sujeitos da pesquisa $(n=12)$ obtidos na avaliação

\begin{tabular}{lcc}
\hline & Masculino & Feminino \\
\hline Comprimento do passo (m) & 46,70 & 36,53 \\
Comprimento da passada (m) & 93,40 & 73,06 \\
Tempo da passada (s) & 10 & 14,16 \\
Velocidade da passada (m/s) & 0,58 & 0,50 \\
Cadência (passos/min) & 76,98 & 84,21 \\
\hline
\end{tabular}

Fonte: Dados da pesquisa.

e Patiño et al. (21-30), esses valores se mostram abaixo dos indivíduos hígidos. Esses autores registraram comprimento da passada $(1,55 ; 1,28 ; 1,32 ; 1,56 ; 1,00$ e 1,39$)$, velocidade $(1,53 ; 1,30 ; 1,16 ; 1,39 ; 1,13 ; 1,50$; $1,03)$ e cadência $(107,115,110,108,120,124)$.

Essa diferença pode ser explicada por fatores como: baixa estatura $(1,53 \mathrm{~m} \pm \mathrm{DP} 0,11)$; sobrepeso (IMC = 26,42 DP $\pm 3,83$ ); frouxidão ligamentar; e pelo pé plano apresentado pela amostra estudada. 
Conforme Filipin, Sacco e Lobo (31), em seu estudo em que foram avaliados indivíduos eutróficos (IMC 15,80) e comparados com indivíduos com sobrepeso (IMC 26,60 ), os sujeitos que tinham sobrepeso apresentavam alteração na distribuição da pressão plantar durante a marcha em todas as regiões do pé, o que pode predispor o aplainamento do arco longitudinal medial, favorecendo alterações no ciclo da marcha.

Correa e Pereira (32) realizaram avaliação da marcha em dois grupos de jovens, um com alteração do arco plantar e outro sem essa alteração, e verificaram que indivíduos com pé plano apresentaram marcha com ritmo imprevisível, hesitação constante e longa antes de passar de um pé de apoio para o outro, não apresentando harmonia nem sincronismo durante o ciclo da marcha, pois o excesso de pronação não permite que a articulação subtalar alcance posição neutra antes da elevação do calcanhar, tornando a base de apoio instável para a propulsão e um equilíbrio ineficiente do corpo sobre a base de suporte.

Em relação à baixa estatura, Byung-Sik, Bang e Bong (33) em seu estudo avaliaram 15 sujeitos com SD, compararam o resultado com 10 indivíduos sem histórico de doença neuromusculoesquelética e constataram que diferenças antropométricas podem influenciar no padrão da marcha.

Esses mesmos autores (33) obtiveram na avaliação da marcha dos indivíduos com SD os seguintes valores: para comprimento de passada $72,8 \mathrm{~cm}$; e para velocidade da passada $69,8 \mathrm{~cm} / \mathrm{seg}$. No item cadência não houve diferença significativa entre os dois grupos, ambos apresentaram média de 114 passos por minuto; em relação à amostra deste estudo, no que se refere a comprimento da passada, percebese que o valor é semelhante ao grupo do sexo feminino aqui avaliado, que apresentou comprimento da passada de $73,6 \mathrm{~cm}$. A velocidade e a cadência, no entanto, diferem de ambos os gêneros, que aqui apresentaram 0,60 m/s (DP $\pm 0,15)$ e 89,35 (DP $\pm 14,33)$, respectivamente.

Entretanto, alguns valores obtidos neste estudo também diferem dos adquiridos por Felício et al. (34), que usou o mesmo método aplicado nesta pesquisa para obter os dados de comprimento do passo e cadência com jovens com e sem SD. Ele observou, então, que os jovens típicos de 15 anos apresentaram comprimento do passo de $62,54 \mathrm{~cm} \pm 6,2$ e os de 20 apresentaram $63,5 \mathrm{~cm} \pm 1,9$; já os jovens com SD apresentaram de $52,8 \mathrm{~cm} \pm 7,2$ e $52,4 \mathrm{~cm} \pm 4,2$, respectivamente. Em nosso estudo os jovens mostraram valores inferiores tanto em relação ao grupo típico quanto ao SD referido pelo autor.

Nesse mesmo estudo (34) a cadência dos jovens com SD foi maior que dos jovens típicos, os com SD de 15 anos tiveram cadência de 120 passos/minuto e os de 20 anos foram 90 passos/minuto. Os jovens típicos da mesma idade apresentaram cadência de 96 passos/minuto e de 60 passos/minuto, respectivamente. 0 valor obtido no presente estudo para cadência foi de 89,35 (DP $\pm 14,33$ ) e vai ao encontro dos valores obtidos para jovens com SD de 20 anos, citados anteriormente, considerando que a média de idade da nossa amostra é de 18 anos $( \pm 2,8)$.

Felício et al. (34) também justifica que, embora haja diferença entre as variáveis obtidas, não são consideradas significativas a ponto de prejudicar o jovem com Down, cuja trajetória do desenvolvimento da motricidade pode ser vista como paralela às crianças típicas, mas de forma mais lenta; justificativa considerável, pois, como já foi exposto em alguns estudos, jovens com SD têm características semelhantes às de jovens hígidos ou, em alguns casos, existem pequenas diferenças.

Outros agravantes encontrados nessa população, que pode alterar a característica da marcha, é a hiperfrouxidão ligamentar $(3,35)$ e a hipotonia muscular (35). Uma menor força muscular pode tornar a marcha mais arrastada pela dificuldade do indivíduo em sustentar o peso, levando a uma compensação pela flexão de joelho (36), fato que pode justificar os valores baixos obtidos para velocidade, pois o indivíduo dispõe de um tempo maior na fase de apoio.

Em nossa amostra os parâmetros avaliados apresentam diferença entre o grupo feminino e o masculino. Estrázulas et al. (36) avaliaram 24 mulheres hígidas com idade média de $25,3( \pm 5,9)$ e registraram para tamanho do passo o valor de $0,62 \mathrm{~m}$ e cadência de 108,03 p/min. Esses valores foram semelhantes ao de Guimarães et al. (27), que avaliaram 20 mulheres com idade média de 23,4 (DP $\pm 1,50$ ) e registraram para tamanho do passo $0,63 \mathrm{~m}$, velocidade de $1,13 \mathrm{~m} / \mathrm{s}$ e cadência de $108 \mathrm{p} / \mathrm{min}$; valores que diferem dos obtidos para a amostra feminina e masculina deste estudo, que foi, para tamanho do passo, de $36,53 \mathrm{~m}( \pm 7,55), 46,70 \mathrm{~m}( \pm 5,99)$ e cadência de 84 , $21 \mathrm{~m}( \pm 7,07)$ e $76,98( \pm 18,40)$, respectivamente.

Chung (25) avaliou 44 sujeitos do sexo masculino com idade média de 27 anos e registrou a média para tamanho do passo de $64,33 \mathrm{~cm}$, comprimento 
da passada de $128,66 \mathrm{~cm}$, velocidade de $116,46 \mathrm{~cm} / \mathrm{s}$ e cadência de 108,26 p/min, valores que diferem da amostra masculina e feminina deste estudo. Contudo, os valores obtidos por Chung (25) vão ao encontro dos valores citados anteriormente por Estrázulas et al. (36) para a amostra feminina.

Com isso, observa-se que indivíduos do sexo masculino e feminino podem apresentar diferenças nos parâmetros da marcha, porém essas diferenças são mínimas ou em alguns casos não existem, devendo considerar as características antropométricas, que já foram abordadas neste estudo como fatores que influenciam nos parâmetros da marcha.

\section{Conclusão}

Os valores obtidos para as variáveis lineares (tamanho do passo e passada, cadência e velocidade da marcha) evidenciados neste estudo demonstraram que jovens com síndrome de Down apresentam déficit na marcha em relação a indivíduos hígidos descritos na literatura.

Sugere-se para futuros estudos investigar o desenvolvimento motor do indivíduo relacionado à estimulação recebida quando criança para identificar possíveis diferenças entre grupos estimulados e não estimulados.

\section{Referências}

1. Nussabaum RL, Mcinnes RR.Thompson \& Thompson: genética médica. Rio de Janeiro: Guanabara Koogan; 2002.

2. Cooley WC, Graham JM. Down syndrome: an update and review for the primary pediatrician. Clin Pediatr. 1991;30(4):233-53.

3. Matos MA. Instabilidade atlantoaxial e hiperfrouxidão ligamentar na síndrome de Down. Acta Ortop Bras. 2005;13(4):165-67.

4. Shumway-Cook A, Woollacott MH. Controle motor: teoria e aplicações práticas. São Paulo: Manole; 2003.

5. Campos D, Santos DCC. Controle postural e motricidade apendicular nos primeiros anos de vida. Fisioter Mov. 2005;18(3):17-7.
6. Viel E. A marcha humana, a corrida e o salto: biomecânica, investigações, normas e disfunções. Barueri: Manole; 2001.

7. Ribas DIR, Dias M, de Marco M, Santos DS. Marcha infantil: uma breve revisão de literatura. Anais da I Jornada Catarinense Multi/Interdisciplinar em Pediatria. Itajaí: UNIVALI; 2005. p. 130-31.

8. Caon G, Ries LGK. Triagem do desenvolvimento motor nos dois primeiros anos de vida. Pediat Moderna. 2003;39(7):248-52.

9. Mancini MC, Silva PC, Gonçalves SC, Martin SM. Comparação do desempenho funcional de crianças portadoras de síndrome de Down e crianças com desenvolvimento normal aos 2 e 5 anos de idade. Arq Neuropsiquiatr. 2003;61(2-B):409-15.

10. Boff J, Caregnato RCA. História oral de mulheres com filhos portadores de síndrome de Down. Rev Bras Enfermagem. 2008;17(3):576-86.

11. Van Der Fits IBM, Otten E, Klip AWJ, Van Eykern LA, Hadders-Algra M. The development of postural adjustments during reaching in 6 to 18 months old infants. Exp Brain Res. 1999;126(4):517-28.

12. Araújo AGS, Scartezini CM, Krebs RJ. Análise da marcha em crianças portadoras de síndrome de Down e crianças normais com idade de 2 a 5 anos. Fisioter Mov. 2007;20(3):79-85.

13. Mark LL, Anson JG. What are "normal movements" in atypical populations? Behavioral Brain Sciences. 1996;19:55-68.

14. Winter DA, Mackinnon CD, Ruder GK, Wieman C. An integrated EMG/biomechanical model of upper body balance and posture during human gait. Prog Brain Res. 1993;97:359-67.

15. Alonso VK, Okaji SS, Pinheiro MT, Ribeiro CM, Souza HP, Santos SS, et al. Análise cinemática da marcha em pacientes hemiparéticos. Rev Fisioter Brasil. 2002; 55:16-23.

16. Sutherland DH, Olshen RA, Biden EN, Wyatt MP. The development of mature walking. Oxford: Mac Keith Press; 1988.

17. Perry J. Análise da marcha: marcha normal. Barueri: Manole; 2005. 
18. Saad M, Battistella LR, Masiero D. Técnicas de análise de marcha. Acta Fisiátrica. 1996;3(2):23-6.

19. Ariani C, Penasso P. Análise clínica cinemática comparativa da marcha de uma criança normal e outra portadora de síndrome de Down na fase escolar (7 a 10 anos). Rev Reabilitar. 2005;26(7):17-23.

20. Almeida CAN, Baptista MEC, Almeida GAN, Ferraz VEF. Obesidade infanto-juvenil: uma proposta de classificação clínica. Pediatria. 2004;26(4):257-67.

21. Winter D. Biomechanics and motor control of human movement. New York: A Wiley Interscience Publication; 1990.

22. Winter D. Biomechanical motor patterns in normal walking. J Mot Behav. 1983;15(4):302-30.

23. Rose J, Gamble JG. Marcha humana. São Paulo: Premier; 1998.

24. Holden MK, Gill KM, Magliozzi MR. Gait assessment for neurologically impaired patients. Phys Ther. 1986; 66(10):1530-9.

25. Chung TM. Avaliação cinética e cinemática da marcha de adultos do sexo masculino. Acta Fisiátrica. 2000;7(2):61-7.

26. Barela AMF, Stolf SF, Duarte M. Biomechanical characteristics of adults walking in shallow water and land. J Electromyogr Kinesiol. 2006;16(3):250-6.

27. Guimarães ECL, Santos SP, Raimundo AKS, Santana LAN, Dini PD, David AC. Estudo da repetibilidade das variáveis espaço-temporais da marcha de indivíduos saudáveis. Fisioter Mov. 2007;20(4):83-90.

28. Ribas DIR, Israel VL, Manfra EF, Araújo CC. Estudo comparativo dos parâmetros angulares da marcha humana em ambiente aquático e terrestre em indivíduos hígidos adultos jovens. Rev Bras Med Esporte. 2007;13(6):371-5.

29. Dini PD, David AC. Repeatability of spatiotemporal gait parameters: comparison between normal children and children with hemiplegic spastic cerebral palsy. Rev Bras Fisioter. 2009;13(3):215-22.
30. Patiño MS, Gonçalves AR, Monteiro BC, Santos IL, Barela AMF, Barela JA. Características cinemáticas, cinéticas e eletromiográficas do andar de adultos jovens com e sem suporte parcial de peso corporal. Rev Bras Fisioter. 2007;11(1):19-25.

31. Filippin NT, Barbosa VLP, Sacco ICN, Lobo da Costa PH. Efeitos da obesidade na distribuição da pressão plantar em crianças. Rev Bras Fisioter. 2007;11(6): 495-501.

32. Correa AL, Pereira JS. Correlação entre a redução dos arcos plantares e as alterações da marcha, equilíbrio e postura em escolares. Rev Bras Ci Mov. 2005; 13(4):47-54.

33. Byung-Sik KMD, Bang DY, Bong-OK KMD. Gait characteristic in Down's syndrome. Gait\&Posture, 1995; 3(2):84. doi: 10.1016/0966-6362(95)93461-K.

34. Felício SR, Gava NM, Zanella RC, Pereira K. Marcha de crianças e jovens com síndrome de Down. ConScientiae Saúde. 2008;7(3):349-56.

35. Soares MPS, Lemos SS, Barros JF. Detecção de características específicas do joelho que podem limitar a atividade física em portadores da síndrome de Down no DF. 2003 [acesso 25 jun. 2010];9(61). Disponível em: http://www.efdeportes.com/encuentra. htm?cx=partner - pub $-7621742700180041 \% 3$ Agat9we-5w32\&cof=FORID\%3A10\&ie=ISO-8859$1 \& \mathrm{q}=$ Detec $\% \mathrm{E} 7 \% \mathrm{E} 3 \mathrm{o}+\mathrm{de}+$ caracter $\%$ EDsticas + espe cificas+do+joelho+que+podem+limitar+a+atividad $\mathrm{e}+\mathrm{f} \%$ EDsica $+\mathrm{em}+$ portadores $+\mathrm{da}+\mathrm{S} \%$ EDndrome $+\mathrm{de}$ +Down+no+DF\&sa=Buscar\#625.

36. Estrázulas JA, Pires R, Santos DM, Stolt LROG, Melo SIL. Características biomecânicas da marcha em crianças, adultos e idosos. 2005 [acesso 25 jun. 2010];10 (88). Disponível em: http://www.efdeportes.com/ efd88/biom.htm.
Recebido: $17 / 10 / 2010$

Received: $10 / 17 / 2010$

Aprovado: $23 / 05 / 2011$

Approved: 05/23/2011 